\title{
Could Pion Electroproduction Be a Test of Current-Current Light-Cone Commutators?
}

\author{
A. Burnel \\ Physique Théorique et Mathématique, Université de Liège - Liège \\ (Lett. Nuovo Cimento, 11, 301 (1974))
}

Two mistakes (which do not however affect our conclusions) have been pointed out to me.

i) In the r.h.s. of eq. (4a), there should be an additional term proportional to

$$
\varepsilon_{\mu \lambda \nu \tau} \partial^{\lambda}\left[\varepsilon\left(x_{0}\right) \delta\left(x^{2}\right)\right] J_{a b}^{\mu \tau}(x, 0) .
$$

Such a term implies a term proportional to $(1 / v) \varepsilon_{\mu \lambda \nu \tau} q^{\lambda} p_{1}^{y} p_{2}^{x}$ in the r.h.s. of eq. $(8 a)$.

ii) A correct treatment of the nucleon spin would imply an additional term of the form $\vec{u}\left(p_{2}\right) \gamma^{\tau} \gamma_{5} u\left(p_{1}\right) B_{30}^{(5) a b}\left(x \cdot p_{1}, x \cdot p_{2}, t, q^{\prime 2}\right)$ in the r.h.s. of eq. $(5 b)$. If such a term modifies the coefficients of $p_{i \mu}$ in eq. $(8 b)$, it does however not imply their vanishing, so that our conclusion is not affected. Because of the first mistake, it cannot however be stated that the coefficient of $\varepsilon_{\mu \nu \lambda \tau} q^{\nu} p_{1}^{\lambda} p_{2}^{\tau}$ in the r.h.s. of eq. (8b) must vanish.

by Società Italiana di Fisica

Proprietà Ietteraria riservata

Direttore responsabile: CARLO CASTAGNOLI

Stampato in Bologna dalla Tipografia Compositori coi tipi della Tipografia Monograf

Questo fascicolo è stato licenziato dai torchi il 20-XII-1974 